\title{
Intégrer la voix des patients au JAMC
}

\author{
Victoria Saigle MSc, Jean Miller PhD BSc inf, Vincent Dumez MSc, Kirsten Patrick MBBCh MSc
}

Citation : CMAJ 2021 July 12;193:E1046-7. doi : 10.1503/cmaj.211092-f

Voir la version anglaise de l'article ici : www.cmaj.ca/lookup/doi/10.1503/cmaj.211092

$\mathbf{L}$ e Groupe JAMC reconnaît depuis longtemps l'importance de la mobilisation des patients dans la production du savoir médical ${ }^{1-3}$. Au cours de l'année écoulée, avec nos patients partenaires, nous avons conçu des politiques et établi des pratiques optimales dans le but d'intégrer la voix des patients dans la gouvernance du JAMC et de refléter leur point de vue dans un plus grand nombre d'articles.

Ce numéro porte sur la participation des patients partenaires à l'exploration ${ }^{4,5}$, à la conception et à l'application de politiques sanitaires axées sur les patients ${ }^{6,7}$, sur l'importance de comprendre leur expérience et celle des proches aidants ${ }^{8}$, et il propose des renseignements pratiques pour les patients qui gèrent leurs problèmes de santé à la maison ${ }^{9}$. Le JAMC a adopté la définition du mot patient des Instituts de recherche en santé du Canada, soit un terme général qui englobe toutes les personnes vivant avec un problème de santé ainsi que les proches aidants, y compris les amis et la famille (https://cihr-irsc.gc.ca/f/48413.html).

Les problèmes de santé ne surviennent pas isolément; les gens les gèrent dans le contexte de leur situation de vie et ils acquièrent ainsi une expertise en la matière. Ces dernières années, l'expertise des patients a contribué à orienter la recherche en identifiant des enjeux d'importance pour eux et qui n'entraient pas en ligne de compte auparavant au moment d'évaluer la réussite de nouveaux traitements (p. ex., la fatigue chez les personnes atteintes de polyarthrite rhumatoïde ${ }^{10}$ ) et les obstacles à l'accès aux soins de santé ou aux traitements (p. ex., problèmes de transport ou schémas posologiques compliqués). En outre, des initiatives communautaires ont permis de rendre certaines stratégies sanitaires plus acceptables et mieux culturellement adaptées ${ }^{11}$. Les équipes de collaboration avec les patients partenaires s'attaquent à des enjeux thérapeutiques qui concernent autant le système de santé que les patients, comme les besoins de soutien de ces derniers pour leur autoprise en charge et la création de processus et d'outils d'admission centralisés qui, tout en répondant aux besoins des patients, ont aussi amélioré le système ${ }^{12-14}$.

Lorsqu'il est question de la mobilisation des patients, il importe de porter attention aux personnes elles-mêmes. Certaines sentent parfois qu'elles peuvent exprimer leur point de vue uniquement si on les y invite, comme le montrent des articles du présent numéro ${ }^{7,8}$. Les personnes qui vivent de la discrimination ou de la stigmatisation et les groupes marginalisés sont moins susceptibles de donner leur opinion sur le système de santé ou de participer à des activités de mobilisation. Des facteurs tels que la connaissance des protocoles de soins ou de recherche, le degré d'instruction, les moyens financiers pour participer sans rémunération, la confiance à l'endroit du système de santé et les contraintes de temps limiteront l'éventail des points de vue entendus. C'est quelque chose que nous avons l'intention de surveiller à l'interne, alors que le JAMC continue de mettre en œuvre ses propres initiatives pour la mobilisation des patients.

Au cours de la dernière année, nous avons intégré des patients partenaires dans la gouvernance du JAMC en les incluant dans nos groupes consultatifs pour co-créer des politiques et prioriser les contenus, et nous les avons soutenus à titre d'auteurs et de pairs-réviseurs. Cela suit son cours. À ce jour, les mesures concrètes qui ont été prises incluent la création de politiques de rémunération pour nos partenaires, la simplification et la flexibilisation des marches à suivre (p. ex., pour la soumission des manuscrits), et la mise en œuvre de mécanismes d'évaluation périodique de nos initiatives de mobilisation des patients.

Ce numéro thématique inaugure un nouveau type d'article à la section "Pratique, Consultation- $360^{\circ}$ ». Ces articles mettront en lumière certains aspects interpersonnels et systémiques des soins de santé rarement abordés dans les autres articles de cette rubrique du $J A M C^{8}$. Chaque Consultation- $360^{\circ}$ comportera une brève histoire de cas et les réflexions personnelles de 2-4 personnes impliquées dans une même consultation clinique; 1 des auteurs sera toujours un patient, un membre de la famille ou un proche aidant. Les réflexions des auteurs mettront en évidence divers points de vue représentés au cours de la consultation.

La mobilisation des patients n'est ni une mode ni une passade. Elle apporte aux soins de santé des points de vue particuliers. Elle favorise la collaboration, la communication et la pratique éthique, et ancre fermement la pratique dans des expériences vécues qui sont très enrichissantes sur le plan du savoir. Au-delà, de l'anecdote, le point de vue des patients permet aux professionnels de la santé, qui travaillent souvent en vase clos, de mieux comprendre le système de santé. Les patients parlent et réfléchissent en termes de «trajectoires de 
vie », tandis que les professionnels de la santé se concentrent ponctuellement sur le problème clinique en cause. Les patients mobilisent naturellement leurs communautés et agissent comme agents d'influence dans la société. Ils apportent des connaissances et des habiletés spécifiques qui sont indispensables à l'innovation médicale. C'est pourquoi la mobilisation des patients au JAMC est essentielle.

Intégrer la voix des patients dans le fonctionnement du JAMC reposera sur un processus itératif. Nous croyons qu'il aura des retombées durables pour notre lectorat, les médecins en exercice, la population canadienne, les chercheurs et le système de santé canadien. Pour les lecteurs peu familiers avec la mobilisation des patients, nous espérons que ce numéro thématique fera la lumière sur quelques-unes des nombreuses façons dont l'implication des patients peut améliorer les soins de santé. Les lecteurs peuvent consulter notre déclaration d'intention pour la mobilisation des patients ici : https://www.cmaj.ca/statement -purpose-patient-engagement.

\section{Références}

1. Patrick K. Realizing the vision of patient-relevant clinical research. CMAJ 2016;188:1063.

2. Patrick K, Kebbe M, Aubin D. A home for patient-oriented research. CMAJ 2018;190:E607.

3. Laupacis A. CMAJ priorities for 2020. CMAJ 2019;191:E1344.

4. Campbell RB, Larsen M, DiGiandomenico A, et al. The challenges of managing diabetes while homeless: a qualitative study using photovoice methodology. CMAJ 2021;193:E1034-41.

5. Nykiforuk CIJ. Engaging patients in research using photovoice methodology. CMAJ 2021;193:E1050-1.

6. Denegri S, Starling B. COVID-19 and patient engagement in health research: What have we learned? CMAJ 2021;193:E1048-9.

7. Buchanan F. How are we going to do this? CMAJ 2021;193:E1052-3.

8. Oxland PR, Barnes SL, Cottrell T, et al. End of life: from chronic rhinosinusitis to a late diagnosis to intensive care. CMAJ 2021;193:E1042-4.

9. Mostarac I, Thomas J, Atzema C. Monitoring blood pressure at home: guidance for Canadian patients. CMAJ 2021;193:E1045.
10. de Wit M, Abma T, Koelewijn-van Loon M, et al. Involving patient research partners has a significant impact on outcomes research: a responsive evaluation of the international OMERACT conferences. BMJ Open 2013;3:e002241.

11. Bharadwaj L. A framework for building research partnerships with First Nations communities. Environ Health Insights 2014;8:15-25.

12. Marlett N, Shklarov S, Marshall D, et al. Building new roles and relationships in research: a model of patient engagement research. Qual Life Res 2015;24: 1057-67.

13. Carr ECJ, Patel JN, Ortiz MM, et al. Co-design of a patient experience survey for arthritis central intake: an example of meaningful patient engagement in healthcare design. BMC Health Serv Res 2019;19:355.

14. Mrklas KJ, Barber T, Campbell-Scherer D, et al. Co-design in the development of a mobile health app for the management of knee osteoarthritis by patients and physicians: qualitative study. JMIR Mhealth Uhealth 2020;8:e17893.

Intérêts concurrents : https://www.cmaj.ca/page/staff

Affiliations : Institut de recherche de l'Hôpital d'Ottawa (Saigle), Ottawa, Ont.; Programme PaCER [Patient and Community Engagement Research - Mobilisation des patients et de la communauté dans le cadre de la recherche] (Miller), Faculté de médecine, Université de Calgary, Calgary, Alb.; Directeur des partenariats communautaires (Dumez), Faculté de médecine, Université de Montréal; Centre d'excellence sur le partenariat avec les patients et le public (Dumez), Faculté de médecine, Université de Montréal, Centre de recherche du CHUM, Montréal, Qc; Unité de soutien SRAP du Québec, Longueuil, Qc; Réseau Québécois COVID - Pandémie (Dumez); responsable, Mobilisation des patients, JAMC, rédactrice en chef par intérim, JAMC (Patrick).

Propriété intellectuelle du contenu : Il s'agit d'un article en libre accès distribué conformément aux modalités de la licence Creative Commons Attribution (CC BY-NC-ND 4.0), qui permet l'utilisation, la diffusion et la reproduction de tout médium à la condition que la publication originale soit adéquatement citée, que l'utilisation se fasse à des fins non commerciales (c.-à-d., recherche ou éducation) et qu'aucune modification ni adaptation n'y soit apportée. Voir : https://creativecommons.org/licenses/by-nc-nd/4.0/deed.fr.

Correspondance : Rédaction du JAMC, editorial@cmaj.ca 